\title{
THE INTERNATIONAL CALENDAR
}

\author{
LYONETTE LOUIS-JACQUES
}

\section{6}

\section{Association of American Law Schools (AALS)}

Annual Meeting: "Empirical Scholarship: What Should We Study and How Should We Study It?"

[includes discussions of LL.M. programs for foreign lawyers and practicing law in different jurisdictions]

Washington, D.C.

January 3-7, 2006

Websites: http://www.aals.org/am2006/ http://www.aals.org/aalscal.html

Note: [was moved from New Orleans, Louisiana, January 4-8, 2006 because of impact of Hurricane Katrina]

\section{American Lawyer Media}

LegalTech $25^{\text {th }}$ Anniversary

New York, NY

January 30 - February 1, 2006

Websites: http://www.almevents.com/r5/cob_page.asp?

category_id $=32941$

http://www.legaltechshow.com/

* Foreign and International Law Librarian and Lecturer in Law, D'Angelo Law Library, University of Chicago Law School.

The compiler welcomes news and information on conferences and other meetings of interest to IALL members. Please contact her via email atllou@midway.uchicago. $e d u$ or letter at D'Angelo Law Library, University of Chicago School of Law, $1121 \mathrm{E}$. $60^{\text {th }}$ St., Chicago, IL 60637, U.S.A.; tel: (773) 702-9612; fax: (773) 702-2889.

The closing date for this column was October 31, 2004. For up-to-date information, check the Calendar at the IALL website at http://www.iall.org. 
Institute of Museums and Library Services (IMLS)

Web-Wise 2006: $7^{\text {th }}$ Annual Institute

February/March 2006?

Website: http://www.imls.gov/pubs/conferences.htm

American Bar Association (ABA)

2006 Midyear Meeting

Chicago, Illinois

February 8-13, 2006

Website: http://www.abanet.org/midyear/2006

European Law Faculties Association (ELFA)

ELFA Annual General Meeting and Conference

Leuven, Belgium

Febraury 9-11, 2006

Website: http://www.elfa-afde.org/html/frameset.html

Rechtswissenshaftliche Fakultät, Universität Wien

IRIS 2006: Internationales Rechtsinformatik Symposion = International Legal Informatics Symposium

February 16-18, 2006

Vienna, Austria

Website: http://www.univie.ac.at/RI/IRIS2006

\section{Juriconnexion}

Assemblée Générale annuelle (Annual General Meeting)

Paris, France

March 2006

Website: http://www.juriconnexion.info/

\section{Practising Law Institute (PLI)}

The Law Library 2006

[focuses on library management]

March 1, 2006 (San Francisco)/March 16, 2006 (New York)

San Francisco, California/New York, NY

Websites: http://www.pli.edu/

http://www.pli/edu/product/program_viewall.asp?ptid=511

Seminar on the Acquisition of Latin American Library Materials

(SALALM) 
SALALM 51: 51 ${ }^{\text {st }}$ Annual Meeting: "Crossing Borders, Latin American Migrations: Collections and Services for/from New Library Users"

Santo Domingo, Dominican Republic

March 19-22, 2006

Websites: http://www.library.cornell.edu/colldev/SALALM51Press

Release.htm

http://www.salalm.org

http://www.library.cornell.edu/colldev/salalmhome.html

\section{Nigerian Association of Law Libraries (NALL)}

2006 Conference

Nigeria

March 20-26, 2006?

\section{Verein Deutscher Bibliothekare (VDB)}

Bibliothekartag in Dresden: "Netzwerk Bibliothek"

[includes the meeting of the Arbeitsgemeinschaft für juristisches Bibliotheksund Dokumentationswesen or AjBD at $h t t p: / / w w w . a j b d . d e /]$

Dresden, Germany

March 20-24, 2006

Website: http://www.bibliothekartag.de

http://www.vdb-online.org

\section{International Studies Association (ISA)}

$47^{\text {th }}$ Annual ISA Convention: "The North-South Divide and International Studies"

San Diego, California, USA

March 22-25, 2006

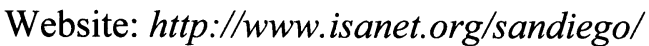

\section{European Institute of Public Administration (EIPA)}

Seminar: Europe on the Internet

Maastricht, The Netherlands

March 23-24, 2006

Website: http://www.eipa.nl/default.htm

\section{Philippine Librarians Association Inc.}

XIIIth Congress of Southeast Asian Librarians (CONSAL) 2006:

"CONSAL at the Crossroads: Challenges for Greater Regional Cooperation" [CONSAL XIII General Conference]

Manila, Philippines

March 25-30, 2006 
Contacts: Antonio M. Santos

Antonio.santos@up.edu.ph

Prudenciana C. Cruz

nanie@nlp.gov.ph

Websites: http://law.upd.edu.ph/ consal/index.html

http://law.upd.edu.ph/ consal/themes.html

http://dlsu.edu.ph/lirary/plai/

\section{American Society of International Law (ASIL)}

ASIL Centennial $-100^{\text {th }}$ Annual Meeting: "A Just World Under Law"

Washington, D.C., USA

March 29-April 1, 2006

Website: http://www.asil.org/Centennial/index.html

http://www.asil.org/events/annualmeeting.html

http://www.asil.org/events/calendar.cfm

http://asil.intracommunities.org/calendar

Vereinigung der juristischen Bibliotheken der Schweiz = Association des bibliothèques juridiques suisses

Jahrestagung $=$ Assemblèe annuelle

Fribourg, Switzerland

March 30-31, 2006

Websites: http://www.stub.unibe.ch/html/jb/rechtsbib/index.html http://www.lawlibraries.ch/

Political Studies Association (PSA)

$56^{\text {th }}$ PSA Annual Conference 2006

University of Reading, UK

April 4-6, 2006

Websites: http://www.psa.ac.uk/

http://www.psa.ac.uk/2005/dfault.htm

Section of International Law, American Bar Association (ABA)

2006 Section Spring Meeting

New York, NY

April 5-8, 2006

Website: http://www.abanet.org/intlaw/meet/home.html\#upcoming

\section{American Bar Association (ABA)}

ABA TECHSHOW 2006: "The World's Premier Legal Technology

Conference \& Expo"

[Burgess Allison will be the keynote speaker] 
Chicago, Illinois

April 20-22, 2006

Websites: http://www.techshow.com/

http://www.abanet.org/techshow/

European Institute of Public Administration (EIPA)

Seminar: European Information

Brussels, Belgium

April 24, 2006

Website: http://www.eipa.nl/default.htm

\section{Texas Library Association (TLA)}

TLA 2006 Annual Conference

Houston, Texas, USA

April 25-28, 2006

Website: $h t t p: / / w w w . t x l a . o r g / c o n f e r e n c e / c o n f . h t m l$

http://www.txla.org/calendar.html\#dates

Canadian Association of Law Libraries / Association Canadienne des Bibilothèques de Droit (CALL / ACBD)

2006 CALL/ACBD Conference: "Four Strong Winds" (Leadership,

Resources, Innovation, and Expectations)

Edmonton Alberta Canada

May 7-10, 2006

Website: http://www.callacbd.ca/

International Association for Social Science Information Service and Technology (IASSIST)

IASSIST 2006: "Data in a World of Networked Knowledge"

Ann Arbor, Michigan

May 22-26, 2006

Website: http://www.iassistdata.org/conferences/

Association of Caribbean University, Research and Institutional Libraries (ACURIL)

ACURIL XXXVI: "Information and Human Rights = Información y

Derechos Humanos = Information et Droits Humains"

Biblioteca Nacional Aruba, Aruba

May 28-June 2, 2006

Website: http://acuril.rrp.upr.edu/index.htm

International Law Association (ILA) 
$72^{\text {nd }}$ Conference

Toronto, Canada

June 4-8, 2006

Websites: http://www.ila-hq.org/html/layout_event.htm http://www.ila-hq.org

\section{American Lawyer Media (ALM)}

LegalTech West Coast 2006

The Westin Bonaventure Hotel, Los Angeles, California June 5-6, 2006

Websites: http://www.almevents.com/r5/display.asp?file= calendar2006.asp

http://www.legaltechshow.com/

\section{Nordic Law Librarians' Group}

Det 7. Nordiske juridiske bibliotekmøte $=7^{\text {th }}$ Nordic Law Librarians' Meeting [triennial conference $-1^{\text {st }}, 1989\left(\right.$ Oslo) $; 2^{\text {nd }}, 1991$ (Copenhagen); $3^{\text {rd }}, 1994$ (Helsingfors); $4^{\text {th }}, 1997$ (Stockholm); $5^{\text {th }}, 2000$ (Oslo); $6^{\text {th }}, 2003$ (Reykjavík)]

Copenhagen, Denmark

June 7-9, 2006

Contact: Lisbeth Rasmussen, University of Copenhagen, Law

Students' Library at the Faculty of Law, Studiestraede 34, DK1455 Copenhagen, Denmark

Tel: 45 35323303; Fax: 4535323313

Email: lisbeth.rasmussen@jur.ku.dk

Website:

http://www.jur.ku.dk/biblioteker/nordiske_juridiske_biblioteks moedel

\section{European Institute of Public Administration (EIPA)}

Seminar: Who's Afraid of European Infromation?

Maastricht, The Netherlands

June 8-9, 2006

Website: http://www.eipa.nl/default.htm

\section{Special Libraries Association (SLA)}

$97^{\text {th }}$ Annual Conference

[includes programs of the SLA Legal Division]

Baltimore, Maryland, USA

June 11-14, 2006

Website: http://www.sla.org/content/Events/index.cfm http://www.slalegal.org 


\section{British \& Irish Association of Law Librarians (BIALL)}

$27^{\text {th }}$ Annual BIALL Study Conference \& Exhibition 2006: "Facing the Front" [includes pre-conference seminar on June 14, 2006; the conference will focus on the themes of leadership, team working and motivation, and cover information literacy, collaboration, and methods for teaching legal research skills]

Brighton, UK

June 15-17, 2006

Website: http://www.biall.org.uk/home.asp

\section{The Center for Computer-Assisted Legal Instruction (CALI)}

$16^{\text {th }}$ Annual CALI Conference for Law School Computing

Nova Southeastern Shepard Broad Law Center, Fort Lauderdale, FL June 15-17, 2006

Websites: http://www2.cali.org/index.php?fuseaction=conference. home http://www.cali.org/

\section{American Library Association (ALA)}

Annual Conference

New Orleans, Louisiana, USA

June 22-28, 2006

Website: http://www.ala.org/events

Australian and New Zealand Society of International Law (ANZSIL) 2006 Annual Conference

Australia

June/July 2006

Website: http://www.law.usyd.edu.au/scigl.anzsil/

\section{Caribbean Association of Law Libraries (CARALL)}

CARALL 2006: $21^{\text {st }}$ Annual Conference

Trinidad \& Tobago

July 2006

Website: http://www.carallnet.org

\section{Law and Society Association (LSA)}

Annual Meeting

Baltimore, Maryland, USA

July 6-9, 2006

Website: http://www.lawandsociety.org/ann_mtg/ 
meeting_lst.htm

http://www.lawandsociety.org/ann_mt_gen.htm

\section{American Association of Law Libraries (AALL)}

$99^{\text {th }}$ Annual Meeting and Conference: "Pioneering Change"

[2006 is the $100^{\text {th }}$ anniversary of the Association; AALL will celebrate its achievements at the annual meeting and throughout the centennial year]

America's Center, St. Louis, Missouri, USA

July 8-12, 2006

Website: http://aallnet.org/events

http://aallnet.org/events/am_locations.asp

\section{International Political Science Association (IPSA)}

$20^{\text {th }}$ IPSA World Congress: "Is Democracy Working?"

Fukuoka, Japan

July 9-13, 2006

Websites: http://www.ipsa.ca/en/about/congresses.asp

http://www.ipsa.ca

http://www.fukuoka2006.com/en

\section{American Society of Comparative Law (ASCL) and International}

Academy of Comparative Law (IACL)

2006 Annual Meeting and XVIIth Congress of the International Academy of Comparative Law

Utrecht, The Netherlands

July 16-22, 2006

Website: http://www.comparativelaw.org/meet.html\#2006

http://www.comparativelaw.org

http://www2.law.uu.nl/priv/AIDC/index1.asp

\section{Southeastern Association of Law Schools (SEALS)}

2006 Annual Meeting

Palm Beach, Florida

July 16-22, 2006

Website: http://www.nsulaw.nova.edu/seals/

\section{American Bar Association (ABA)}

Annual Meeting

Honolulu, Hawaii, USA

August 3-9, 2006

Website: http://www.abnet.org/annual/futmeet.html 
Australian Law Librarians Group (ALLG), American Association of Law Libraries (AALL), British and Irish Association of Law Librarians (BIALL), Canadian Association of Law Libraries (CALL), and the New Zealand Law Librarians Group Inc. (NZLLG)

Joint Study Institute (JSI)

Oxford, England, UK

August 10-14, 2006

Contact: Loyita Worley, Chair of JSI Organising Committee, Head of Library, Information \& KM

Richards Butler, Beaufort House, 15 St Botolph Street, London EC3A 7EE, United Kingdom

Phone: +44 (0) 2077725747

Fax: +44 (0) 2075395240

Email: lmw@richardsbutler.com

Websites: http://www.biall.org.uk/jsi2006.asp

International Federation of Library Associations and Institutions (IFLA) World Library and Information Congress: $72^{\text {nd }}$ IFLA General Conference and Council

Seoul, The Republic of Korea

August 20-24, 2006

Website: http://www.ifla.org/IV/ifla72/index.htm

\section{University Association for Contemporary European Studies (UACES)}

UACES $36^{\text {th }}$ Annual Conference and $11^{\text {th }}$ Research Conference: "Visions of Europe: Key Problems, New Trajectories"

Limerick, Ireland

August 31-September 2, 2006

Website: http://www.uaces.org/Limerick.htm

\section{American Political Science Association (APSA)}

$102^{\mathrm{d}}$ Annual Meeting

San Francisco, California, USA

August 31-September 4, 2006

Website: http://www.apsanet.org/mtgs/future.cfm

\section{European Institute of Public Administration (EIPA)}

Seminar: European Information

Brussels, Belgium

September 4, 2006

Website: http://www.eipa.nl/default.htm 
International Association of Law Libraries (IALL)

$25^{\text {th }}$ Course on International Law Librarianship and Conference

St. Petersburg, Russia

September 10-14, 2006

Contact: Halvor Kongshavn, Bergen University Library, Law Library, N-5020 Bergen, Norway

Phone: +4755 589525

Fax: +4755 589522

Email: halvor.kongshavn@ub.uib.no

Website: http://www.iall.org

http://www.lla.ru/

\title{
International Bar Association (IBA)
}

IBA Conference 2006

Chicago, Illinois, USA

September 17-22, 2006

Website: http://www.ibanet.org

http://www.ibanet.org/images/downloads/Confs\%202005.pdf

\section{Australian Library and Information Association (ALIA)}

ALIA 2006 Biennial Conference: click06: Create, Lead, Innovate, Connect, Knowledge

Perth Convention Centre, Perth, Australia

September 19-22, 2006

Website: http://conferences.alia.org.au/alia2006 http://conferences.alia.org.au

\section{International Association of Procedural Law (IAPL)}

"The Reception and Diffusion of Civil Procedure Law in the Global Society from the Aspect of Legal Assistance and Legal Education"

Ritsumeikan University, Kyoto, Japan

September 20-22, 2006

Website: http://www.uni-regensburg.de/Fakultaeten/Jura/gottwald/ internassprocedurallaw/

\author{
New Zealand Law Librarians Inc. (NZLL) \& Australian Law Librarians \\ Group (ALLG) \\ 2006 ALLG/NZLL Joint Conference: "Southern Currents 2006" \\ Melbourne University, Legal Resource Centre, Melbourne, Australia \\ September 27-29, 2006 \\ Websites: http://www.nzll.org.nz/ \\ http://www.nzll.org.nz/events.cfm?folderid $=20$
}


http://www.allg.asn.au/

\section{Canadian Council on International Law (CCIL)}

Conference 2006

Ottawa, Canada

October 2006

Website: http://www.ccil-ccdi.cal

American Branch of the International Law Association (ABILA)

2006 International Law Weekend

New York, NY

October 2006

Website: http://www.ambranch.org/

\section{T.M.C.Asser Instituut}

Asser College Europe Training Course for Legal Information Specialists

[Application deadline: 30 June 2006]

The Hague, The Netherlands

October/November 2006

Websites: $h t t p: / / w w w . a s s e r . n l / i n s t i t u t e /$ categorieen/index/asp? ${ }_{-} \_n r=6$ http://www.asser.nl/institute/index.asp

\section{Illinois Library Association (ILA)}

2006 ILA Annual Conference

Navy Pier, Chicago, Illinois

October 3-6, 2006

Websites: http://www.ila2006.org/

http://www.ila.org/events/calendar.htm

http://www.ila.org/events/index.htm

\section{European Institute of Public Administration (EIPA)}

Seminar: Europe on the Internet

Maastricht, The Netherlands

October 5-6, 2006

Website: http://www.eipa.nl/default.htm

\section{Information Today, Inc. (ITI)}

Internet Librarian 2006

October 23-25, 2006

Website: http://www.infotoday.com/conferences.shtml 
New York Library Association (NYLA)

2006 NYlA Conference \& Trade Show

Saratogo Springs, NY

November 1-4, 2006

Website: http://www.nyla.org/index.php?page_id $=122$

American Society for Information Science \& Technology (ASIS\&T)

2006 Annual Meeting

Austin, Texas, USA

November 3-9, 2006

Website: http://www.asis.org

\section{European Institute of Public Administration (EIPA)}

Seminar: Keep Ahead with European Information in the Enlarged Europe Information and Communication Strategies

Maastricht, The Netherlands

November 30-December 1, 2006

Website: http://www.eipa.nl/default.htm 